\title{
Non-Invasive Ventilation (NIV) a Study of Junior Doctor Competence
}

\author{
J.O.M. Plumb*, M. Juszczyszyn and G. Mabeza
}

Royal Derby Hospital, Uttoxeter Road, Derby, DE22 3NE, UK

\begin{abstract}
Background: There is currently no standardisation to undergraduate or postgraduate teaching in the use of NIV in the UK.

Methods: A questionnaire-based survey (see appendix) was handed out to all foundation doctors (year 1 and year 2) at Derbyshire Hospitals NHS Foundation Trust between January 2008 and April 2008. NIV was provided in the emergency department, on the acute respiratory ward and in intensive care. Foundation trainees did not work in intensive care at the time of the study. The study primarily focused on NIV in the setting of acute exacerbations of chronic obstructive pulmonary disease (COPD). The questionnaire was designed to primarily test the subject's knowledge of the physiological principles of NIV including its indications and contradictions and some of the common problems surrounding its use.

Results: There were 47 respondents from a cohort of 105 foundation trainees. 12 (26\%) of doctors had received no undergraduate teaching/training about NIV. $33(70 \%)$ rated the training they did have as poor. Knowledge surrounding the use of NIV was poor, only $15 \%$ had positive knowledge scores. $28(60 \%)$ of the doctors who had used NIV stated that they had low confidence when using it. Interestingly of the doctors who scored highly 6 of the 7 had done a respiratory medicine job. A positive score on the questionnaire was directly related to having spent time working in respiratory medicine $(\mathrm{p}<0.0001)$.

Conclusions: There is no standardisation to undergraduate or postgraduate teaching in the use of NIV in the UK. Foundation level trainees have poor knowledge of NIV and low confidence in using it. Working in a respiratory job with an acute NIV service increases knowledge and confidence. Being competent to administer and manage a patient on NIV is an important competence for junior medical staff. At present great improvements need to be made in the delivery of training to achieve this competence.
\end{abstract}

Keywords: NIV, COPD, Postgraduate education, Acute care skills, Simulation.

\section{INTRODUCTION}

Non-invasive ventilation (NIV) in the management of acute type II respiratory failure in chronic obstructive pulmonary disease (COPD) patients represents one of the major technical advances in respiratory care over the last decade with a reduction in mortality of approximately $50 \%$ demonstrated in studies [1]. The National Institute for Health and Clinical excellence (NICE) in the UK has therefore recommended that this service be available in all hospitals admitting patients such patients and currently over $90 \%$ of UK admitting hospitals offer an acute NIV service [2] .While there are comprehensive national clinical guidelines on the use of NIV aimed at setting standards for the optimal delivery of acute NIV in the UK, concerns have been raised with regards to the training and competency assessment of staff at the fore-front of acute NIV service delivery [3] .

\section{CURRENT PROBLEMS WITH NIV EDUCATION}

The current guidelines do set out standards for the set up and use of NIV in the UK, but there is little current literature concerning the training and competency assessment of staff involved in this service $[4,5]$.There is no comprehensive guideline specifically designed for the training of staff

*Address correspondence to this author at the Royal Derby Hospital, Uttoxeter Road, Derby, DE22 3NE, UK; Tel: +44 1332 340131;

E-mail: jamieplumb@doctors.org.uk undertaking NIV delivery. This has the potential of leading to widespread variations in clinical practices across the UK [5]. Given the fact that even at a conservative estimate, a typical district general hospital serving a population of 250 000 would expect to treat more than 70 patients per year, it is imperative to ensure that all staff involved in NIV care are not only adequately trained but that competency in NIV provision is properly assessed.

We therefore set out to investigate the current knowledge and competence amongst foundation level trainees (junior doctors in their first 2 years post graduation) expected to come across a patient on NIV during their work, particularly 'on call' when working outside of conventional working hours. Foundation level trainees in the UK do 2 years (Foundation year $1 \mathrm{FY} 1$, and Foundation year 2 FY) made up of 6x 4 monthly rotations, 4 months at the very least must include internal medicine and this will involve seeing acutely unwell medical patients. We specifically aimed to ascertain how much prior training the junior doctors had received on NIV, their level of knowledge of the clinical indications for NIV, and their level of confidence in troubleshooting problems in patients already established on NIV. At the time of the study (2008) all foundation doctors in the UK were required to do a medical job for at least 4 months of their foundation training to include medical 'on call work'.

Foundation level trainees involved in the study would be expected to meet patients on NIV or requiring NIV either in 
the emergency department or on the acute respiratory ward. This primarily, but not exclusively involved patients with acute exacerbations of COPD. Patients with respiratory failure requiring non-invasive ventilation for other reasons i.e. pneumonia, acute pancreatitis, sepsis would be managed on intensive care and foundation doctors would therefore not be involved in their management unless they were established on NIV in the emergency department.

\section{METHODS}

\section{Subjects and Settings}

\section{Setting- a UK Based Foundation Trust Hospital}

A questionnaire-based survey (see appendix 1) was handed out to all foundation doctors (year 1 and year 2) at Derbyshire Hospitals NHS Foundation Trust between January 2008 and April 2008. The two foundation years are the years immediately after graduation from medical school in the UK; these junior doctors were the ones expected to come across a patient on acute NIV at some point during their rotation as all foundation doctors in the trust were required to complete a minimum of 4 months in a medical speciality by the end of their programme during which they would spend time on an 'unselected on call rota.'

The exercise was carried out during the breaks in central teaching sessions. The questionnaire took approximately 15 minutes to complete. The authors observed the participants as they filled out the questionnaire to ensure there was no conferring between participants.

\section{Questionnaire}

An initial pilot was carried out during the design of the questionnaire on 10 middle grade and senior house officers to highlight any ambiguous questions, which were subsequently removed.

The questionnaire was designed to primarily test the subject's knowledge of the physiological principles of NIV including its indications and contradictions and some of the common problems surrounding its use. The questionnaire also tested knowledge and understanding of the possible ethical dilemmas that arise when using NIV. The questionnaire also explored the qualitative aspect of NIV use by using scales to rate soft variables such as confidence and comfort with the technique.

The knowledge score was calculated as follows. Questions 6-13 were used to form the basis of the score. Questions $7,8,9,11,12$ and 13 were simply right or wrong so 1 point was awarded for a correct answer. Question 6 listed options, 1 point was awarded for the correct answer but 1 point was taken away for an incorrect answer. Extra points were given if they stated relative or absolute. Question 10 had a list of patient choices, again 1 point was awarded if the answer was correct but a point was deducted if the answer was incorrect. It was therefore deemed very easy to score positively with minimal knowledge of NIV.

The authors gave due consideration to the ethical dimensions of this anonymous questionnaire and no concerns were identified. The questionnaire was optional and completion was taken as consent to participate.

\section{Statistical Methods}

Fisher's exact test was used to compare doctors who had positive knowledge scores and those that had negative knowledge scores with if they had worked in a respiratory medicine job or not.

\section{RESULTS}

There were 47 respondents from a cohort of 105 foundation trainees. These were made up of 26 females and 21 males. 28 were FY2 doctors and 19 were FY1.

Only 4 doctors had no experience of medical on call work. $12(26 \%)$ of doctors had received no undergraduate teaching/training about NIV. $33(70 \%)$ rated the training they did have as poor, only $1(2 \%)$ rated the teaching they had received as good and a further $1(2 \%)$ had never heard of NIV.

Since graduating $24(51 \%)$ stated that they had received training/teaching in the use of NIV. $16(34 \%)$ of doctors had experience of using NIV, 41 (88\%) of these doctors had used

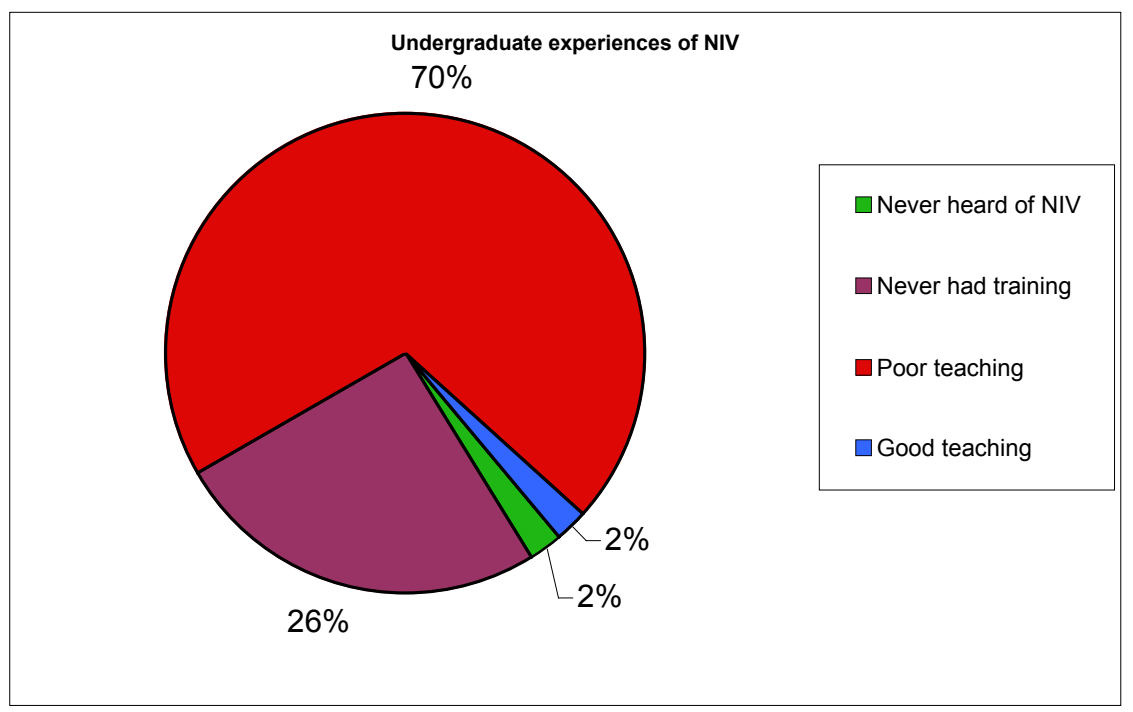

Fig. (1). Undergraduate experiences of teaching on NIV. 


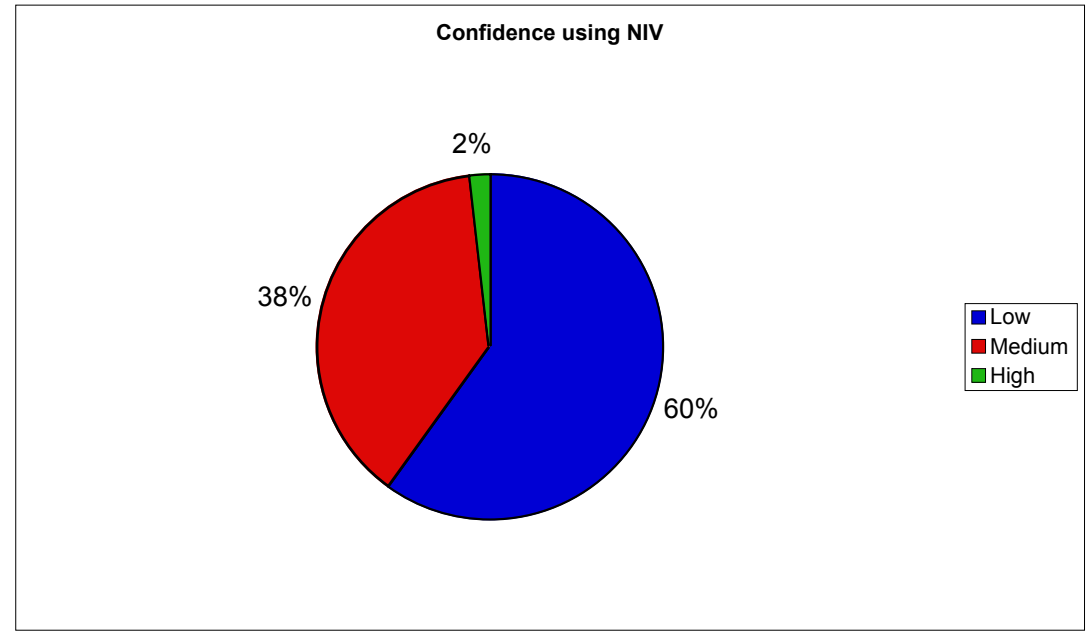

Fig. (2). Confidence using NIV.

NIV whilst on call. The remaining $6(12 \%)$ of doctors had worked on a respiratory ward with acute NIV beds. Only 4 doctors surveyed stated that they felt that they had 'lots of experience' with NIV management, all of these doctors had worked on a respiratory ward for at least 4 months.

$28(60 \%)$ of the doctors who had used NIV stated that they had low confidence when using it with 18 (38\%) having medium confidence and only $1(2 \%)$ stating they had high confidence. When asked how doctors felt when managing patients on NIV not a single doctor felt confident and over $50 \%$ admitted to 'being out of their depth' and worried by the prospect. $95 \%$ admitted to being unable to set up and operate an NIV machine to establish a patient on NIV in an emergency. The study design did not require that the $5 \%$ claiming to be competent in setting up NIV demonstrate their competence.

Knowledge surrounding the use of NIV was poor. Doctors' knowledge was tested using a variety of formats (see appendix 1). Total scores were worked out with candidates loosing marks for incorrect answers. As the knowledge levels were so low they were analysed in 2 groups, positive scores and negative scores. Only $15 \%$ actually had a positive score. Interestingly of the doctors who scored highly 6 of the 7 had done a respiratory medicine job $\mathrm{p}<0.0001$. This was found to be statistically significant using Fisher's exact test the 2 tallied $\mathrm{p}$ value was $<0.0001$ inferring a direct relationship between increased knowledge of NIV and time spent working in an acute NIV service.

Respondents were split when asked if they felt that their undergraduate training had focused more on the care of the 'unwell' surgical patient.

\section{DISCUSSION}

Undergraduate medical training has changed significantly in the last 10 years and will almost certainly continue to change $[6,7]$. In the UK for instance the Foundation one year (first year after formal qualification) is set to become an extension of medical school, in part due to the European Working Time Directive (EWTD) which limits the hours a

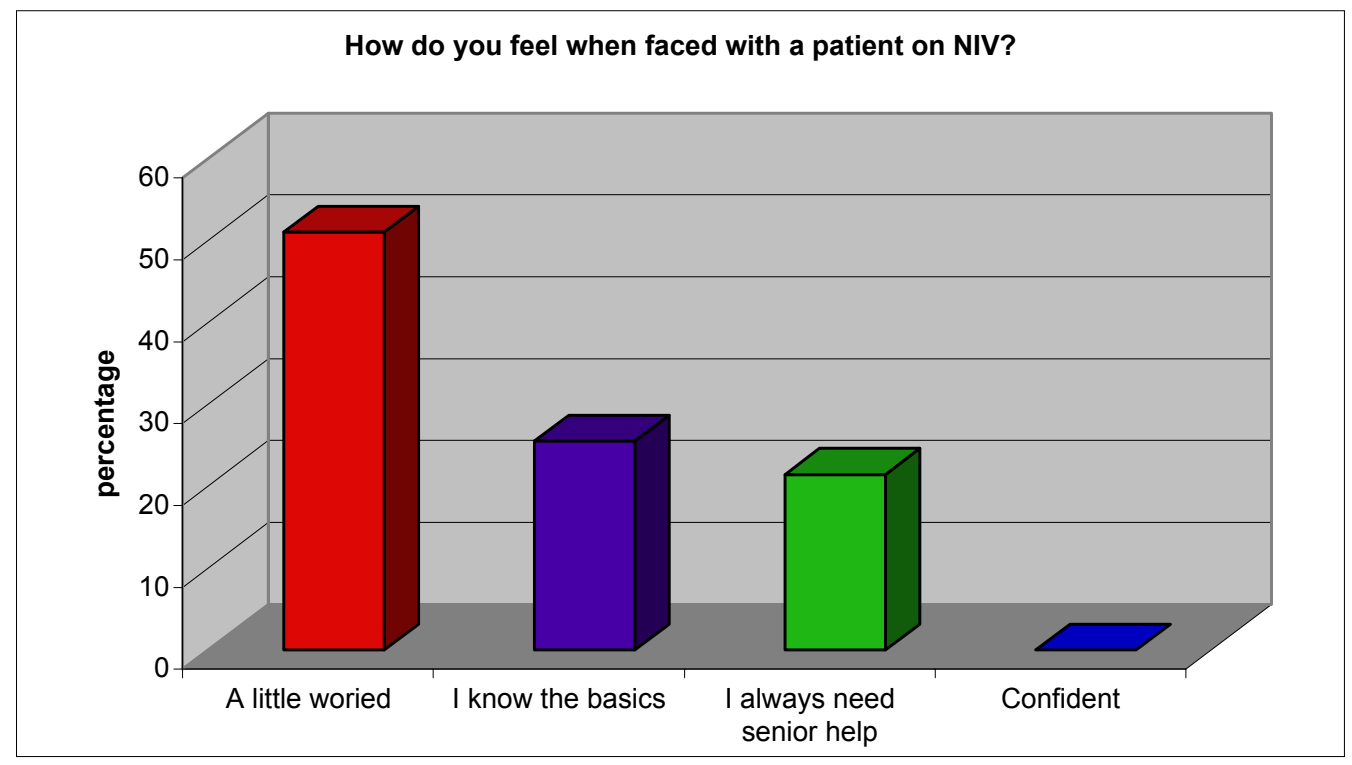

Fig. (3). How doctors felt when asked to look after a patient on NIV. 


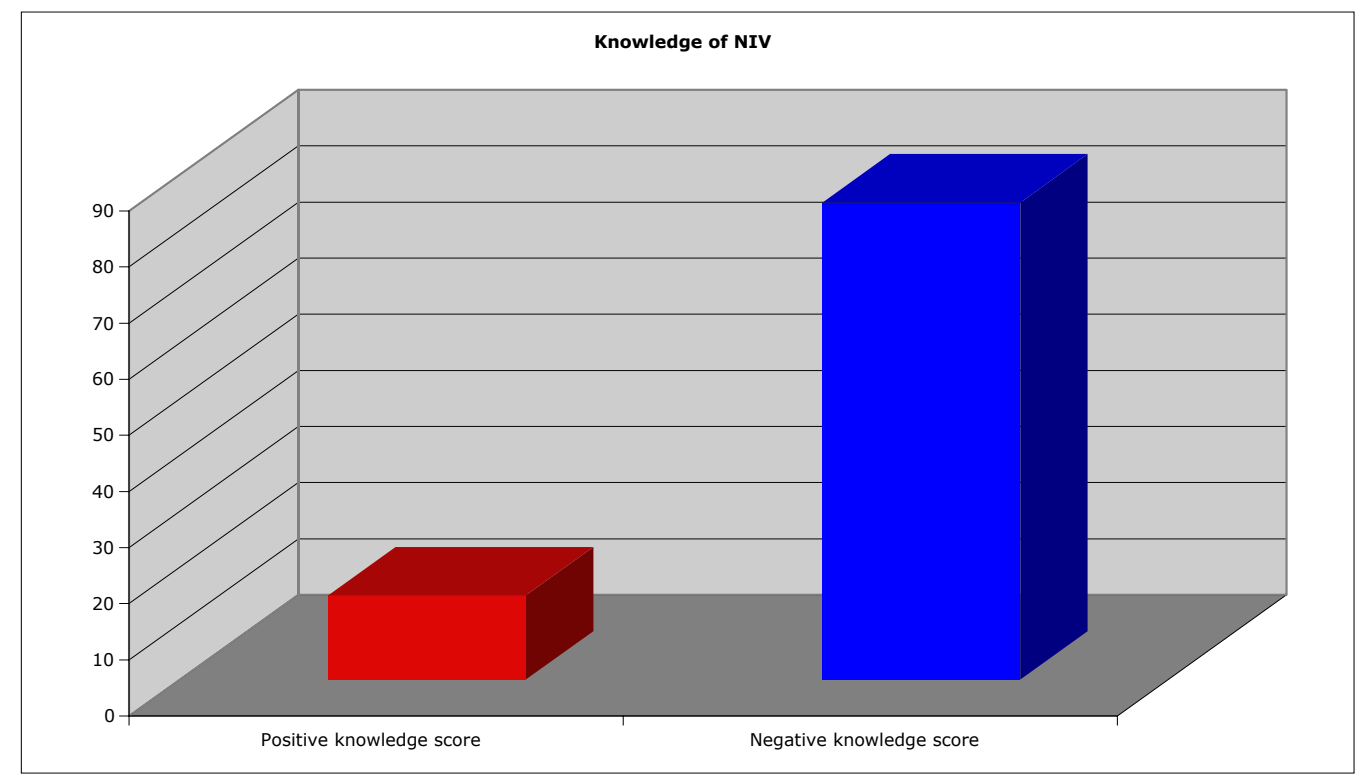

Fig. (4). Knowledge of NIV.

junior doctor can work to 48 hours a week, but also with the continued drive to protect and support newly qualified doctors in a system centred around a competency based assessment model. Such a model rightly places emphasis on a practitioner demonstrating competency in a procedure before practising it independently and thereby reduces the chances of a junior doctor coming across a very unwell patient and being expected to manage them with 'remote senior input'. The hospital at night program recently rolled out across much of the UK, relies on a nurse fielding calls and directing them to an appropriately qualified doctor thus reducing the amount of calls a junior doctor might receive at night which they would need to re-direct to a more appropriate senior grade of doctor.

The gap between knowledge and practice easily becomes apparent when junior doctors are faced with patients requiring 'equipment-based treatment' such as NIV.

While the average UK medical graduate finishes their training with a remarkably transferable set of similar skills, it may be argued that the focus in medical training may be on preparing students for their final examinations, and that the value of the knowledge or skills set at the end of training may be of limited value in day to day clinical practice.

There has indeed been a drive in recent years to try to teach emergency clinical skills and important non-clinical skills to medical students often in the form of simulationbased training $[8,9]$. We believe that NIV as a therapy lends itself well to this kind of training. In our study, despite most respondents receiving some undergraduate teaching and with over half of them receiving post graduate teaching on NIV it was perhaps surprising that practical knowledge levels were still quite poor. Confidence in using NIV was found to be low and half of the cohort admitted to 'feeling out of their depth' when dealing with a patient on NIV. Working in a respiratory job with an acute NIV service increases knowledge $(\mathrm{p}<0.0001)$ and confidence.

With $92 \%$ of foundation trainees recruited having experienced on call medical work and $34 \%$ of them having had to look after a patient requiring NIV the authors concluded that the subject was highly relevant to the studied cohort. It would be interesting to compare confidence and knowledge of other topics (for example how to transcutaneously pace a patient) that are commonly taught on generic courses such as Adult Life Support (ALS) (which all foundation trainees must undertake).

Was the poor knowledge a result of poor teaching? $72 \%$ had either no, or poor undergraduate teaching. It is unclear if this correlation can be made but the lack of teaching was clearly a significant problem. This has already been highlighted by the BTS 'A training programme for the provision of an NIV service should provide a combination of knowledge based learning supported by clinical experience in the workplace.' [3]

It was felt that low confidence came from lack of practical experience with little or no support from senior trainees on the background of a non-existent or thin knowledge base. It was no surprise that the $2 \%$ who had stated that they had 'high confidence' had both done a respiratory medicine job. Part of the problem stems from senior doctors (outside of acute, respiratory medicine or ICU settings) not having a good understanding of its use.

Whilst medicine still has much to learn from industries such as aviation [10] with regard to such things as human factors training, the uptake on simulation training despite its considerable costs has been fairly widespread. Courses such as ALERT $^{\circledR}$ (Acute Life-threatening Events--Recognition and Treatment) [11] (Portsmouth) and Immediate Life Support (ILS) are now compulsory for many medical undergraduates. The shift in experience away from ward based learning to problem based group working and seminar based teaching puts an even greater emphasis on the need for such courses to be delivered in the best way. It was felt so important that the Acute Care Undergraduate Teaching (ACUTE) Initiative was established to ensure that every UK medical student developed these important core competencies of which there are 71 according to ACUTE [12]. 
The ability of such courses and simulation based teaching days to actually improve clinical care is beyond the scope of this paper. However embracing it in the same way as junior doctors have been forced to embrace competency-based assessments will ultimately probably raise standards and there is a growing body of evidence to support this [13].

NIV and the knowledge required to teach the finer points of its use lend itself well to simulation based practical courses. With the number of hours spent 'on call' decreasing and the length of training shortening it becomes ever more pressing to integrate such topics into generic courses and to teach them in a standardised way. There is a wealth of evidence to support this type of algorithm teaching and all of the major life support courses have adopted this style. Traditionalists might argue that it erodes the ability of doctors to make decisions and that algorithms don't leave room for flexibility, but evidence shows that algorithm based learning improves competence and safety. In stressful scenarios human error occurs and an immense body of literature testifies to the value of practices based on principles designed to compensate for human cognitive failings. These include, for example, standardization, simplification, and use of protocols and checklists [14]. In the case of NIV it would form a base of knowledge that could then be expanded upon in the clinical setting. All foundation trainees in the cohort studied had simulation based training days as part of their development during the foundation programme. None however involved the use of NIV machines or scenarios with patients requiring NIV.

\section{WHAT THIS STUDY ADDS}

- There is no standardisation to undergraduate or postgraduate teaching in the use of NIV in the UK.

- Foundation level trainees have poor knowledge of NIV and low confidence in using it.
- Working in a respiratory job with an acute NIV service increases knowledge and confidence.

- $\quad$ Being competent to administer and manage a patient on NIV is an important competence for junior medical staff. At present great improvements need to be made in the delivery of training to achieve this competence.

\section{LIMITATIONS OF THIS STUDY}

The relatively small sample size may have skewed the results. We only looked at foundation trainees from one UK trust although this represented at least 9 different medical schools. It is unclear if there would be regional variations in knowledge. Although knowledge levels correlated well with confidence levels in our cohort it must be recognised that there is an inevitable amount of subjectivity when analysing ones confidence.

\section{CONCLUSION}

There is no standardisation to undergraduate or postgraduate teaching in the use of NIV in the UK. Foundation level trainees in our study had poor knowledge of NIV and low confidence in using it. Working in a respiratory job with an acute NIV service increases knowledge $(\mathrm{p}<0.0001)$ and confidence.

It remains to be seen if improved teaching would lead to increased confidence and ultimately better patient care but we have shown that training needs to improve for effective provision of NIV amongst UK foundation level trainees.

Further research should focus on the application of integrated knowledge and practical skills workshops on improving competence, knowledge and confidence with regard to NIV. One way to do this would be to integrate an NIV scenario into simulation training days and to include it in acute life support courses.

\section{APPENDIX}

\section{Non-Invasive Ventilation (NIV) Questionnaire}

This is not a test; please try to be as honest as possible. It has been perceived that this is a very weak area in the undergraduate curriculum.

What is your current grade?:

F1

F2

(Please circle and write current speciality, if you have ever done medical on-call work please indicate)

I have done medical on-call work in a place that provides an acute NIV service

(Please circle): Yes No

1. How would you rate your undergraduate teaching on Non-Invasive Ventilation (NIV) (Please circle most applicable):

1. I don't know what NIV is (I have never heard of it) 2. I didn't get any

3. Poor

4. Reasonable

5. Good

6. Excellent

(please write any comments)

2. Since starting work (post grad) have you had any guidance/teaching on the use of NIV? (Please circle) If yes please write what i.e. formal/informal?

Yes No 
3. Since starting work how much experience have you had with NIV?

(Please circle) 1 = none, $10=\mathrm{I}$ have done respiratory job and feel very confident using it.

$\begin{array}{llllllllll}1 & 2 & 3 & 4 & 5 & 6 & 7 & 8 & 9 & 10\end{array}$

Only answer question 4 if you answered 2 or above for question 3

4. How confident are you using NIV (please circle) $1=$ no confidence, $10=$ very confident.

$\begin{array}{llllllllll}1 & 2 & 3 & 4 & 5 & 6 & 7 & 8 & 9 & 10\end{array}$

5. How do you feel when managing a patient on NIV? (Please circle)

1. Out of my depth, honestly a little worried

2. A little worried but know the basics

3. I am ok but feel like I need to always run things by my senior

4. I feel confident

5. Completely in control (don't need to ask anything)

6. Circle any contraindications for using acute NIV on a respiratory ward. If you can, state if they are relative or absolute: (please circle more than one if necessary)

- GCS of 5

- Patient with COPD is acidotic and hypoxic

- Asthmatic with type 2 resp failure now with a silent chest

- Patient refusing NIV

- $\mathrm{COPD}$ pH $7.36 \mathrm{PCO}_{2} 5, \mathrm{PaO}_{2} 10$

- Recent facial surgery

- Patient is vomiting

- $\mathrm{PaO}_{2}$ of 3.4

- Pneumothorax un-drained

- Hypercapnic respiratory failure due to chest wall deformity

- Weaning from tracheal intubation

7. True or false; Before NIV is started in a patient a decision about tracheal intubation must be made: (Please circle)

True False

8. Would you be able to teach a nurse how to set up an NIV machine in an emergency? (Please circle)

Yes No

9. What does IPAP stand for? (Write below)

10. Which of the following patients might benefit from acute NIV? (Circle those who would benefit)

1. Known COPD age 68, known type 2 respiratory failure before presents $\mathrm{SOB}$ pH 7.36, $\mathrm{PaO}_{2} 6.7, \mathrm{PCO}_{2} 5.9$ on 2 litres via nasal specs.

2. Known COPD age 79, known type 2 respiratory failure before presents $\mathrm{SOB} \mathrm{pH} 7.37, \mathrm{PaO}_{2} 8.2, \mathrm{PCO}_{2} 8.9$ on 1 litre via nasal specs.

3. Known Asthma age 33, presents acutely SOB, $\mathrm{PEFR}<50 \%$ predicted, $\mathrm{pH} 7.28, \mathrm{PaO}_{2} 8.9, \mathrm{PCO}_{2} 11.3$ on non re-breathe mask 15L.

4. Myotonic dystrophy age 56, $\mathrm{SOB}$ unwell with pneumonia, $\mathrm{pH} 7.32, \mathrm{PaO}_{2} 10, \mathrm{pCO}_{2} 6.9$ not on oxygen.

5. Known COPD, presents unwell, drowsy $\mathrm{pH} 7.29, \mathrm{PaO}_{2}$ 7.1, $\mathrm{PCO}_{2} 8.9$ on 2 litres via nasal specs.

6. COPD anxious, $\mathrm{SOB}$ comes in gasping for breath $\mathrm{pH} 7.33, \mathrm{PaO}_{2} 9.1, \mathrm{PCO}_{2} 11.1$ currently on 15 litres non re-breathe in A\&E.

11. From the list above which patient most worries you?

12. How long after making a change to NIV settings should you be taking an ABG? (Please circle)

5 mins $\quad 15-20$ mins $\quad 50$ mins $\quad 75$ mins

13. Which of the above patients probably doesn't need NIV but simple measures might dramatically help? 
14. Do you think that undergraduate training has focused more on the unwell surgical patient? Do you think that junior doctors (F1\&F2 specifically) are better at treating the unwell surgical patient? (Please circle)

Yes

No

\section{Please give any comments on NIV and your experiences with using it or being taught how to use it:}

\section{REFERENCES}

[1] Ram FS, Picot J, Lightowler J, Wedzicha JA. Non-Invasive positive pressure ventilation for treatment of respiratory failure due to exacerbations of chronic obstructive pulmonary disease. Cochrane Database Syst Rev 2004; (3): CD004104.

[2] NICE: NICE Chronic Obstructive Pulmonary disease: national clinical guideline on management of chronic obstructive pulmonary disease in adults in primary and secondary care. Thorax 2004; 57 (Suppl 1): 192-211.

[3] Committee BTSSoC: BTS Guideline: non-invasive ventilation in acute respiratory failure. Thorax 2002; 57: 192-211.

[4] British Thoracic Society RCoP, The Intensive Care Society: the use of non-invasive ventilation in the management of patients with chronic obstructive pulmonary disease admitted to hospital with acute type II respiratory failure (with particular reference to Bilevel positive pressure ventilation). October 2008.

[5] Price LC, Lowe D, Hosker HS, et al. National COPD Audit 2003: impact of hospital resources and organisation of care on patient outcome following admission for acute COPD exacerbation. Thorax 2006; (61): 837-42.

[6] Towle A. Continuing medical education: changes in health care and continuing medical education for the 21st century. Br Med J 1998; 316: 301-4.

[7] Prideaux D. Managing change in medical education. Triannual newsletter produced by the centre for development of teaching and learning 2004; vol.8 issue3.
[8] Krüger A, Gillmann B, Hardt C, Döring R, Beckers SK, Rossaint R. Teaching non-technical skills for critical incidents: Crisis resource management training for medical students. Anaesthesist 2009; 58(6): 582-8.

[9] Grenvik A, Schaefer JJ 3rd, DeVita MA, Rogers P. New aspects on critical care medicine training. Curr Opin Crit Care 2004; 10(4): 233-37.

[10] Ziv A, Wolpe PR, Small SD, Glick S. Simulation-based medical education: an ethical imperative. Acad Med 2003; 78(8): 783-8.

[11] The ALERT course teaches healthcare staff how to anticipate, recognise and prevent critical illness at an early stage. [http://www.alert-course.com/]

[12] Perkins GD, Barrett H, Bullock I, et al. The Acute Care Undergraduate Teaching (ACUTE) Initiative: consensus development of core competencies in acute care for undergraduates in the United Kingdom. Intensive Care Med 2005; 31(12): 162733 .

[13] Issenberg SB, McGaghie WC, Hart IR, et al. Simulation technology for health care professional skills training and assessment. JAMA 1999; 282(9): 861-6.

[14] Leape LL, Berwick DM, Bates DW. What practices will most improve safety? evidence-based medicine meets patient safety. JAMA 2002; 288(4): 501-7.

(C) Plumb et al.; Licensee Bentham Open

This is an open access article licensed under the terms of the Creative Commons Attribution Non-Commercial License (http://creativecommons.org/licenses/by-nc/3.0/) which permits unrestricted, non-commercial use, distribution and reproduction in any medium, provided the work is properly cited. 